Volume 8. No. 4, April 2020

International Journal of Emerging Trends in Engineering Research

Available Online at http://www.warse.org/IJETER/static/pdf/file/ijeter03842020.pdf

https://doi.org/10.30534/ijeter/2020/03842020

\title{
Dominant Local Tetra Patterns: A New Feature Descriptor for Face Recognition
}

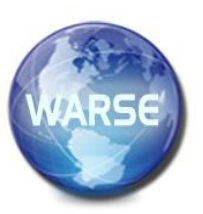

ABSTRACT

Local binary pattern (LBP) and local ternary pattern (LTP) of the gray-level difference of the connection between the identified pixel and its nearby neighbours are encoded based on vertical and horizontal directions using the $\mathrm{n}^{\text {th }}$ order local derivatives. In our proposed approach Dominant local tetra patterns (LTrPs) the $\mathrm{n}^{\text {th }}$ order $\mathrm{LTrP}$ is computed using $\mathrm{n}^{\text {th }}$ order horizontal and vertical local derivatives. The efficiency and the effectiveness of our proposed algorithm for face recognition is calculated and analysed by combining it with the Difference of Gaussian. In order to find the strength of the descriptor various images under different lighting, facial expression and pose are tested. The experimental results show that the proposed approach provides a good representation and advanced of face recognition rates than LBP and its by-products.

Key words: Difference of Gaussian (DoG), Local Binary Pattern (LBP), Local Tetra Patterns (LTrPs), Discrete Wavelet Transform (DWT), Gabor Transform (GT), omplex Wavelet Transform (CWT).

\section{INTRODUCTION}

In the field of pattern recognition face recognition system become popular due to its wide applications in research. Face recognition system application is widely used in human computer interaction security, law enforcement, credit card identification, automated video surveillance and entertainment [1,2]. The features in the face are the most common biometric parameter used by human brain to identify an individual.

Face recognition become critical due to varied lighting, outdoor, indoor, time etc. These limitations associated with face recognition is solved by various methods, most of the methods focus only on single problem and not concentrate on other problems. The face recognition method is classified into appearance based method and feature based method [3]. In appearance based method the image of the whole face is considered and represented, the problem here is in the appearance based method vectors cannot be compared directly so the problem is solved by using reduction technique. The Second method is feature based method, commonly using feature based methods are LBP [4,5], gaussian mixture model and markov model and elastic bunch graph method. These methods provide good result over computation faults of illumination, expression, pose, occlusion and localization. The key objective of this paper is to suggest a good, efficient and accurate descriptor to extract facial features for face recognition. For applications such as computer vision and pattern recognition texture analysis has been extensively used. Wavelet correlogram [6] is the concept proposed by Moghaddam et al. as per his concept using genetic algorithm for face recognition application the performance can further be improved by optimizing the quantization thresholds. Nowadays wide attention is given on Feature texture classification it is a branch of texture analysis and it is appropriate for face identification. Ahmadian et al. have used the discrete wavelet transform (DWT) for texture classification [7]. The DWT can extract only three directional (horizontal, vertical, and diagonal) information from face image. The directional limitations are addressed by Gabor transform (GT) [8,9], rotated wavelet filters, Difference of Guassian (DoG), GT rotated CWFs [10], and rotational invariant complex wavelet filters and have been proposed for texture analysis.

\subsection{Face Image Feature Descriptor}

For face recognition one of the effective texture operator is Local Binary Pattern (LBP), here each pixels of face image are labelled by binary numbers. This is done by thresholding each pixel from its neighbourhood pixel. Due to its discriminative control and computational ease LBP texture operator becomes the most popular approach for texture analysis. For texture analysis LBP approach results good due to the usually differing statistical and structural models. The important properties of LBP operator is its robustness to gray-scale changes due to illumination and other factors and computational simplicity which enable to identify images in real time. Zhang et al. proposed local derivative patterns (LDPs) [11] for face recognition, in which he consider LBP as non-directional first-order local patterns collected from the first-order derivatives and extended the same approach for $\mathrm{n}^{\text {th }}$ order LDPs The LBP has also been used for texture segmentation, background modelling, detection, shape localization, interest region description, and biomedical image retrieval. The different methods of LBP and the LDP 
in the open works cannot sufficiently address the range of appearance variations that commonly occur for free natural images due to aging, expression, illumination, poses, partial occlusions, etc. To deal this problem, the local ternary pattern (LTP) [12] has been introduced for face recognition under different lighting conditions. The edge information's are extracted by LBP, LDP and LTP descriptors, information's are coded only in two directions ie: positive or negative directions. The performance is improved by distinguishing the edges in more directions. Based on this observation local tetra patterns (LTrPs) for face recognition is proposed.

\subsection{Main Contribution}

In this paper, n-order LTrP is proposed and the edge information's are calculated for horizontal and vertical derivatives based on the path of pixels. In LDP's the direction of each pixel in face image is calculated by making use of 0 to 90 derivatives. With analysing the performance of the combination of dual tree complex wavelet transform and LTrP, It is noticed that the proposed method produce good experiment result for face images when compare to LBP, LDP and LTP methods. Alike LDP, when we compare our technique with LBP, LBP is consider as a nondirectional first-order local pattern named first-order LTrP.

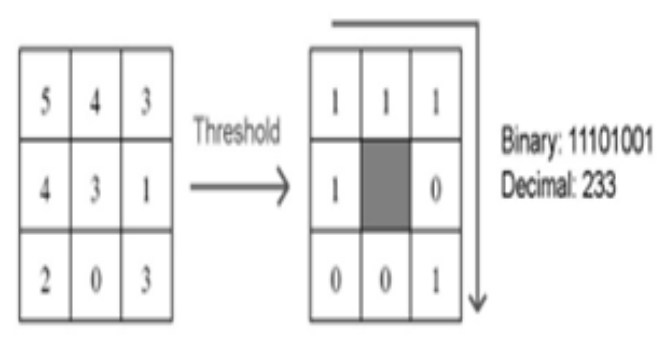

Figure 1: Local Binary Pattern

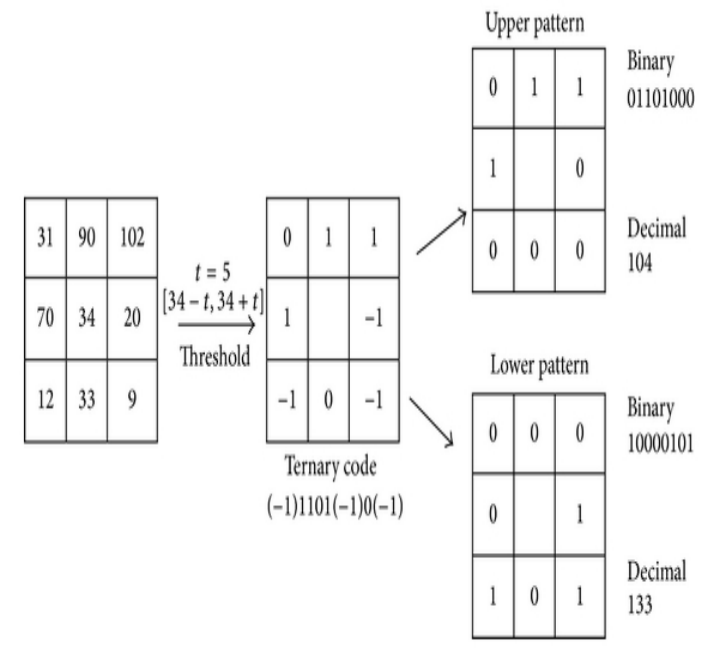

Figure 2: Local Ternary Pattern
Figure 1. shows how the Binary conversions of pixels are made using LBP and LTP operators. In LTP, the upper and lower binary patterns obtained are further coded by retaining 1 and substituting 0 for 1 and 0 for upper pattern and substituting 1 with 1 and 0 for 1 and 0 for Lower pattern.

This paper is organized in such a way that: Section I, gives a short review of the face image feature descriptor and its related work. In Section II various descriptors like LBP, LDP, LTP, and LTrP are discussed. The rest of the paper briefs the idea of multiscale feature extraction, projected system framework, matching, experimental results and finally, conclude by giving short summary of this paper and related future possible work.

\section{RELATED WORK}

\subsection{Local Binary Pattern}

Ojale et al., in 1996 introduced the LBP operator for texture classification. According to Ojale et al the LBP value is computed for a center pixel in an image is by comparing its gray value with its neighbours, this is shown in Figure 1, using equation (1) \& (2)

$$
\begin{aligned}
& \operatorname{LBP}_{\mathrm{P}, \mathrm{R}}=\sum_{\mathrm{k}=0}^{n} 2^{(p-1)}: \mathrm{f}_{1}\left(\mathrm{~g}_{\mathrm{p}}-\mathrm{g}_{\mathrm{c}}\right) \\
& \mathrm{f}_{1}(\mathrm{x})=\left\{\begin{array}{l}
1, x \geq 0 \\
0, \text { else }
\end{array}\right.
\end{aligned}
$$

where $g_{c}$ and $g_{p}$ represent the gray value of the center pixel and gray value of its neighbors, $\mathrm{P}$ signify the number of neighbours, and $\mathrm{R}$ is the neighbourhood radius.

\subsection{Local Ternary Pattern}

Local Ternary Pattern is the extended version of LBP introduced by Tan and Triggs. The gray values in the width zone are quantized to zero and those above and below are quantized to 1 . The three-valued function $\left(x, g_{c}, t\right)$ replaced the indicator by a three-valued code called the LTP, this is shown in Figure 2, using equation (3) for $\mathrm{x}=g_{y}$.

$$
f_{1}\left(\mathrm{x}, \mathrm{g}_{\mathrm{c}}, \mathrm{t}\right)=\left\{\begin{array}{l}
+1, x \geq g_{e}+t \\
0,\left|x-g_{c}\right|<t \\
-1, x \leq g_{c}-t
\end{array}\right.
$$

\subsection{Local Derivative Pattern}

Local Derivative Pattern was proposed by Zhang et al. for face recognition. Zhang et al. considered LBP as a nondirectional first-order local pattern operator and he extended first-order to higher orders ( ${ }^{\text {th }}$-order) and named it as LDP is shown in Figure 3. The LDP encompasses more 
discriminative features in detail as compared to LBP. Local derivative pattern (LDP) captures face features in four directions: $0^{\circ}, 45^{\circ}, 90^{\circ}$ and $135^{\circ}$ and encode it based on local derivative variations and concatenate the result as a $32-$ bit binary string. The first-order derivative $I_{\alpha}^{\prime}(\mathrm{X})$ for a pixel in all the four directions ie: $0^{\circ}, 45^{\circ}, 90^{\circ}$, or $135^{\circ}$ is calculated in a $3 \times 3$ area around it, where $\alpha=0^{\circ}, 45^{\circ}, 90^{\circ}$, and $135^{\circ}$. For a local region $\mathrm{I}(\mathrm{Z})$ if $\mathrm{X}_{0}$ is the central point and $X_{i}$ 's are the neighbours of $X_{0}$ and $i=1,2, \ldots, 8$, then the firstorder derivatives at $\mathrm{X}=\mathrm{X}_{0}$ this is shown in Figure 3 .

$$
\begin{aligned}
& I_{\mathrm{o}}^{\mathrm{f}}\left(\mathrm{X}_{0}\right)=\mathrm{I}\left(\mathrm{X}_{0}\right)-\mathrm{I}\left(\mathrm{X}_{4}\right) \\
& I_{45^{\prime \prime}}{ }^{\prime \prime}\left(\mathrm{X}_{0}\right)=\mathrm{I}\left(\mathrm{X}_{0}\right)-\mathrm{I}\left(\mathrm{X}_{3}\right) \\
& \boldsymbol{z}_{90}^{\prime a}\left(\mathrm{X}_{0}\right)=\mathrm{I}\left(\mathrm{X}_{0}\right)-\mathrm{I}\left(\mathrm{X}_{2}\right) \\
& I_{135}^{\prime}=\left(\mathrm{X}_{0}\right)=\mathrm{I}\left(\mathrm{X}_{0}\right)-\mathrm{I}\left(\mathrm{X}_{1}\right)
\end{aligned}
$$

\begin{tabular}{|c|c|c|c|c|}
\hline${ }_{9} \mathrm{X}$ & ${ }_{10}^{X}$ & ${ }_{11}^{X}$ & ${ }_{12}^{X}$ & ${ }_{13} \mathrm{X}$ \\
\hline${ }_{24} \mathrm{X}$ & $X$ & ${ }_{2} \mathrm{X}$ & ${ }_{3} \mathrm{X}$ & ${ }_{14} \mathrm{X}$ \\
\hline${ }_{23} \mathrm{X}$ & $\begin{array}{l}{ }_{8} X \\
\end{array}$ & $\begin{array}{ll} & X \\
0\end{array}$ & ${ }_{4} \mathrm{X}$ & ${ }_{15} \mathrm{X}$ \\
\hline${ }_{22} X$ & $\begin{array}{l}7_{7} \\
\end{array}$ & $6^{X}$ & $5_{5} \mathrm{X}$ & ${ }_{16} X$ \\
\hline${ }_{21} \mathrm{X}$ & $\begin{array}{r}{ }_{20} \mathrm{X} \\
\end{array}$ & $\begin{array}{l}{ }_{19} \mathrm{X} \\
19\end{array}$ & ${ }_{18} \mathrm{X}$ & $\mathrm{X}$ \\
\hline
\end{tabular}

Figure 3: Example of LDPs representation.

For the calculation of $\mathrm{n}^{\text {th }}$-order LDP, the $(\mathrm{n}-1)^{\text {th }}$-order derivatives should be calculated along the directions $0^{\circ}, 45^{\circ}$, $90^{\circ}$ and $135^{\circ}$, and is denoted as $I_{\mathrm{\alpha}}^{(n-1)}\left(\mathrm{g}_{\mathrm{c}}\right) \mid \alpha=0^{\circ}, 45^{\circ}$, $90^{\circ}, 135^{\circ}$. Finally the $\mathrm{n}^{\text {th }}$-order LDP can be calculated using equation 8 and 9 .

$$
\begin{array}{r}
L D P_{\alpha}^{n}\left(\mathrm{~g}_{\mathrm{c}}\right)=\sum_{p=1}^{p} 2^{(p-1)} \mathrm{X}_{z}\left(I_{\alpha}^{(n-1)}\left(\mathrm{g}_{\mathrm{c}}\right),\right. \\
\left.I_{\alpha}^{(n-1)}\left(\mathrm{g}_{\mathrm{p}}\right)\right)
\end{array}
$$

where $\quad \mathrm{p}=8$

$$
f_{2}(\mathrm{x}, \mathrm{y})= \begin{cases}1, \text { if } x_{\mathrm{r}} y \leq 0 \\ 0, & \text { else }\end{cases}
$$

\subsection{LTrPs}

The local texture spatial structure is determined by the center gray pixel $g_{c}$ along the direction $0^{\circ}$ and $90^{\circ}$ using the firstorder derivative for an image $\mathrm{I}$ and is represented as $I_{0}^{1}, \mathrm{~g}_{\mathrm{h}}$ and $g_{v}$ represent the horizontal and vertical neighbourhoods of $\mathrm{g}_{\mathrm{c} . .}$ The first-order derivatives at the center pixel $\mathrm{g}_{\mathrm{c}}$ is represented in equation 10 and 11.

$$
\begin{aligned}
& I_{0}^{f}=\left(\mathrm{g}_{\mathrm{c}}\right)=\mathrm{I}\left(\mathrm{g}_{\mathrm{h}}\right)-\mathrm{I}\left(\mathrm{g}_{\mathrm{c}}\right) \\
& I_{90^{\circ}}^{\prime}\left(\mathrm{g}_{\mathrm{c}}\right)=\mathrm{I}\left(\mathrm{g}_{\mathrm{v}}\right)-\mathrm{I}\left(\mathrm{g}_{\mathrm{c}}\right)
\end{aligned}
$$

The center pixel directions are calculated using equation 12 .

$$
\begin{aligned}
& I_{D i r}^{\prime}\left(\mathrm{g}_{\mathrm{c}}\right)=\left\{\begin{array}{l}
1_{,} I_{0^{\circ}}^{\circ}(\mathrm{gc}) \geq 0 \text { and } I_{90^{\circ}}(\mathrm{gc}) \geq 0 \\
2, I_{0}^{\prime}(\mathrm{gc})<0 \text { and } I_{90^{\circ}}^{\prime}(\mathrm{gc}) \geq 0
\end{array}\right. \\
& I_{D i r}^{\prime}\left(\mathrm{g}_{\mathrm{c}}\right)=\left\{\begin{array}{l}
3, I_{0^{\circ}}^{\prime}(\mathrm{gc})<0 \text { and } I_{90^{\circ}}^{\prime}(\mathrm{gc})<0 \\
4, I_{0}^{\prime}(\mathrm{gc}) \geq 0 \text { and } I_{90^{\circ}}^{\prime}(\mathrm{gc})<0
\end{array}\right.
\end{aligned}
$$

From the above equation (12), it is clearly noted that there are four possible values for an image ie: each center pixel can be either be 1,2,3, or 4 directions. The second-order derivative of $\operatorname{LTrP}^{2}\left(\mathrm{~g}_{\mathrm{c}}\right)$ is represented in equation 13 and 14

$$
\begin{aligned}
& \operatorname{LTrP}^{2}\left(\mathrm{~g}_{\mathrm{c}}\right)= \\
& \left\{\mathrm{f}_{3}\left(I_{\text {Dir }}^{f}\left(\mathrm{~g}_{\mathrm{c}}\right), I_{\text {Dir }}^{f}\left(\mathrm{~g}_{1}\right)\right), \mathrm{f}_{3}\left(I_{\text {Dir }}^{l}\left(\mathrm{~g}_{\mathrm{c}}\right), I_{\text {Dir }}^{f}\left(\mathrm{~g}_{2}\right)\right), \ldots,\right. \\
& \left.\mathrm{f}_{3}\left(I_{D i r}^{f}\left(\mathrm{~g}_{\mathrm{c}}\right), I_{D i r}^{p}\left(\mathrm{~g}_{\mathrm{p}}\right)\right)\right\}\left.\right|_{\mathrm{p}=8} \\
& \mathrm{f}_{3}\left(I_{D i r}^{\prime}\left(\mathrm{g}_{\mathrm{c}}\right), I_{D i r}^{l}\left(\mathrm{~g}_{\mathrm{p}}\right)\right) \\
& = \begin{cases}0_{F} & I_{D i r}^{p}\left(g_{c}\right)=I_{D i r}^{p}\left(g_{p}\right) \\
I_{D i r}^{p}\left(g_{p}\right) & \text { else }\end{cases}
\end{aligned}
$$

From the above equation (13) and (14) a 8-bit tetra pattern is obtained for each center pixel. Based on the direction of each center pixel all patterns into four parts. Lastly, three binary patterns are obtained by converting the tetra patterns on all four directions.Suppose if " 1 " be the direction of center pixel $I_{D i r}^{\prime}\left(g_{e}\right)$ obtained using (12); then, $\mathrm{LTrP}^{2}$ then the three binary patterns will be as follows:

$$
\begin{aligned}
& \left.\mathrm{LTrP}^{2}\right|_{\text {Direction=2,3,4 }} \\
& =\left.\sum_{p=1}^{p} 2^{(p-1)} \mathrm{X} f_{4}\left(\operatorname{LTr}^{2}\left(\mathrm{~g}_{\mathrm{c}}\right)\right)\right|_{\text {Direction=2,3,4 }}
\end{aligned}
$$

$$
\begin{aligned}
& \left.f_{4}\left(\operatorname{LTrP}^{2}\left(\mathrm{~g}_{\mathrm{c}}\right)\right)\right|_{\text {Direction }=\phi} \\
& = \begin{cases}1, \text { if } \operatorname{LTrP}^{2}\left(g_{\theta}\right)=\emptyset \\
0, & \text { else }\end{cases}
\end{aligned}
$$

where $\emptyset=2,3,4$.

Likewise, center pixels other three directions tetra patterns will be converted to binary patterns to get $12(4 \mathrm{X} 3)$ binary patterns.

Guo et al. proved sign and magnitude components provide improved result for texture classification which are not 
noticed in any individual component. Since sign component extracts more useful information so Guo et al. combines sign LBP with magnitude LBP for texture classification.

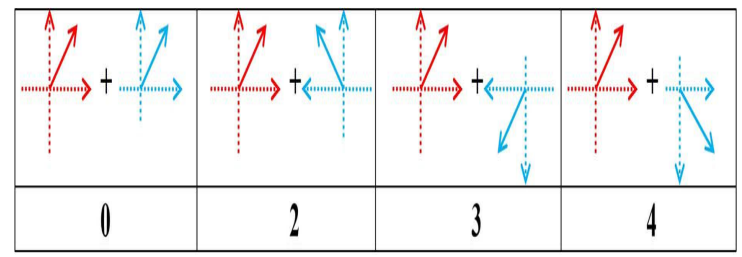

Figure 4: Calculation of tetra pattern bits for the centerpixel direction " 1 " using the direction of neighbors. Direction of (red) the center pixel and (cyan) that its neighborhood pixels.

It was proved by Guo et al. that the combination of sign and magnitude components provide better evidence, that are not obvious in any one individual component although the sign component extracts more useful information as compared with the magnitude component. This motivated us to propose the $13^{\text {th }}$ binary pattern by using magnitudes of horizontal and vertical first-order derivatives using

$$
\begin{aligned}
& \mathrm{M}_{\mathrm{I}}^{1}\left(\mathrm{~g}_{\mathrm{p})}=\sqrt{\left(I_{\mathrm{o}}^{-1}\left(g_{p}\right)\right)^{2}+\left(I_{90}^{1}\left(g_{p}\right)\right)^{2}}\right. \\
& \mathrm{LP}=\Sigma_{p 1}^{p} 2^{(p-1)} \times f_{1}\left(M_{I_{(g)}^{1}}-\left(M_{I_{i g}^{1}}\right)\right)
\end{aligned}
$$

A local pattern with $\mathrm{P}$ neighbourhoods has $2^{\mathrm{P}}$ combinations of LBPs are possible, resulting in the feature vector length of $2^{\mathrm{P}}$. Here for feature vector the combinational cost is very high. So, uniform patterns are considered to reduce the computational cost. The uniform pattern refers to the uniform appearance pattern that has limited discontinuities in the circular binary representation. In this paper those patterns that have less than or equal to two discontinuities in the circular binary representation are referred to as uniform patterns, and the remaining patterns for a given query image would be $\mathrm{P}(\mathrm{P}-1)+2$ are the possible uniform patterns $\mathrm{P}=8$ can be considered.

After identifying the local pattern PTN (the LBP, the LTP, the LDP, or the 13-binary-pattern form LTrP), the whole image is represented by building a histogram using the input image size

$$
\begin{aligned}
& \mathrm{H}_{\mathrm{S}}(\mathrm{l})=\frac{1}{N_{1} X N_{2}} \sum_{j=1}^{N_{1}} \sum_{k=1}^{N_{2}} f_{5}(\mathrm{PTN}(\mathrm{j}, \mathrm{k}), \mathrm{l}) \\
& \mathrm{f}_{5}(\mathrm{x}, \mathrm{y})=\left\{\begin{array}{ll}
1, & \text { if } x=y \\
0, & \text { else }
\end{array} \quad \mathrm{l} \in[0, \mathrm{P}(\mathrm{P}-1)+2](19)\right.
\end{aligned}
$$

is represented by $\mathrm{N}_{1} X \mathrm{~N}_{2}$. Figure 4 illustrates the possible Local pattern transitions resulting in an LTrP for direction ' 1 ' of the center pixel. The LTrP is coded to " 0 " when it is equal to the direction of center pixel, otherwise coded in the direction of neighbourhood pixel. Using the same analogy, LTrPs are calculated for center pixels having directions 2, 3 and 4. For generating a tetra pattern, the bit is coded with the direction of neighbor when the direction of the center pixel and its neighbor are different, otherwise " 0 ." For the magnitude pattern, the bit is coded with " 1 " when the magnitude of the center pixel is less than the magnitude of its neighbor, otherwise " 0 ." An example of the second order LTrP computation resulting in direction " 1 " for a center pixel marked with red has been illustrated in Figure 5. When the first-order derivative is applied in horizontal and vertical directions to the neighbourhood pixel " 8 ", direction " 3 " and magnitude " 9.2 " is obtained.

Since the direction of the center pixel and the direction obtained from the neighbourhood pixel are not same, the value " 3 " is assigned to the corresponding bit of the LTrP. It can be seen that the magnitude of the center pixel is " 6 ", which is less than the magnitude of neighbourhood pixel. Hence, the value " 1 " is assigned to the corresponding bit of the magnitude pattern. Similarly, the remaining bits of the LTrP and the magnitude pattern for the other seven neighbors are computed resulting in the tetra pattern " 3034 0320 " and the magnitude binary pattern " 1111000101 ". After coding the tetra pattern, three binary patterns are

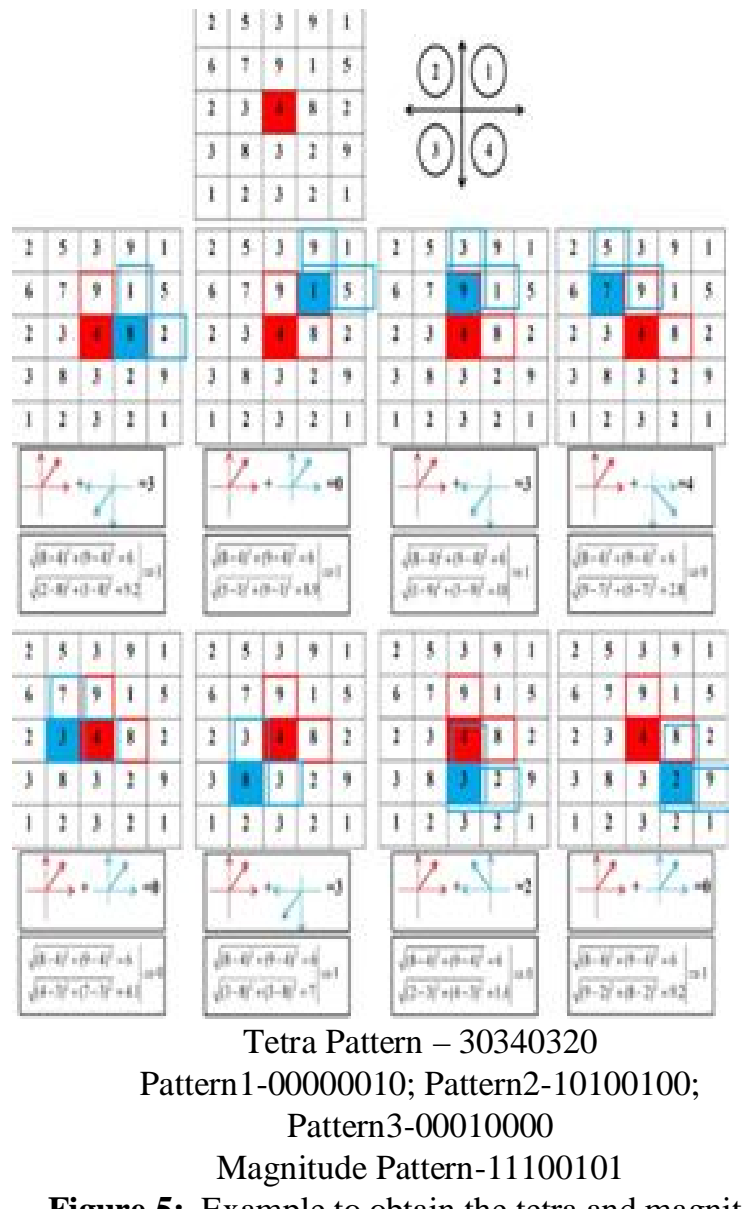

Figure 5: Example to obtain the tetra and magnitude patterns. 
separated as follows. Referring to the generated LTrP, the first pattern is obtained by keeping " 1 " where the tetra pattern value is " 2 " and " 0 " for other values, i.e., "0 00000 010 ". Similarly, the other two binary patterns "1 01010010 0 " and "0 $00 \begin{array}{llllll}0 & 0 & 0 & 0 & 0 \text { " are computed for tetra pattern values }\end{array}$ "3" and "4", respectively. In the same way, tetra patterns for center pixels having directions 2,3 , and 4 are computed. Thus, with four tetra patterns, 12 binary patterns are obtained. The $13^{\text {th }}$ binary pattern is obtained from the magnitude of the first-order derivatives.

\subsection{Nth-Order LTrPs}

To calculate the third-order tetra pattern, initially, the second-order derivatives along horizontal and vertical

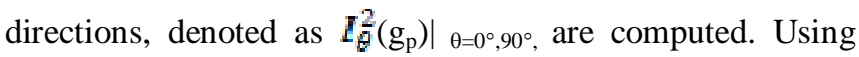
these derivatives, the third-order tetra pattern $\operatorname{LTrP}^{3}\left(\mathrm{~g}_{\mathrm{c}}\right)$ is defined as

$$
\begin{aligned}
& \operatorname{LTrP}^{3}\left(\mathrm{~g}_{\mathrm{c}}\right)=\left\{\mathrm { f } _ { 3 } \left(I_{\text {Dir }}^{2}\left(\mathrm{~g}_{\mathrm{c}}\right),\left(I_{\text {Dir }}^{2}\left(\mathrm{~g}_{1}\right)\right),\right.\right. \\
& \mathrm{f}_{3}\left(I_{\text {Dir }}^{2}\left(\mathrm{~g}_{\mathrm{c}}\right),\left(I_{\text {Dir }}^{2}\left(\mathrm{~g}_{2}\right)\right),\right. \\
& \ldots \ldots,\left.\mathrm{f}_{3}\left(I_{\text {Dir }}^{2}\left(\mathrm{~g}_{\mathrm{c}}\right),\left(I_{\text {Dir }}^{2}\left(\mathrm{~g}_{\mathrm{p}}\right)\right)\right\}\right|_{\mathrm{p}=8}
\end{aligned}
$$

The general formulation for the nth order LTrP can be defined by using (n-1)th-order derivatives in horizontal and vertical directions $\left.I_{\theta}^{n-1}\left(\mathrm{~g}_{\mathrm{p}}\right)\right|_{\theta=0^{\circ}, 90^{\circ}}$ as

$$
\begin{aligned}
& \operatorname{LTrP} \mathrm{p}^{\mathrm{n}}\left(\mathrm{g}_{\mathrm{c}}\right)=\left\{\mathrm { f } _ { 3 } \left(I_{\text {Dir }}^{n-1}\left(\mathrm{~g}_{\mathrm{c}}\right),\left(I_{\text {Dir }}^{n-1}\left(\mathrm{~g}_{1}\right)\right),\right.\right. \\
& \mathrm{f}_{3}\left(I_{\text {Dir }}^{n-1}\left(\mathrm{~g}_{\mathrm{c}}\right),\left(I_{\text {Dir }}^{n-1}\left(\mathrm{~g}_{2}\right)\right),\right. \\
& \left.\ldots \ldots \mathrm{f}_{3}\left(I_{\text {Dir }}^{n-1}\left(\mathrm{~g}_{\mathrm{c}}\right),\left(I_{\text {Dir }}^{n-1}\left(\mathrm{~g}_{\mathrm{p}}\right)\right)\right\}\right|_{\mathrm{p}=8}
\end{aligned}
$$

The higher order LTrPs have the capability to extract more detailed information as compared with lower order LTrPs. However, it has been observed that the second-order LTrP provides better performance as compared with higher order LTrPs. This is primarily because higher order LTrPs are more sensitive to noise.

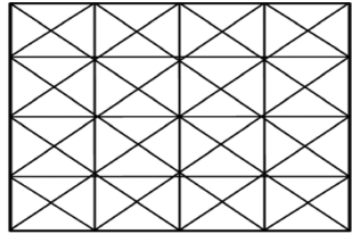

Original Image

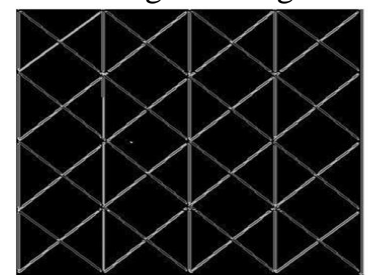

$2^{\text {nd }}$ order LDP in 0

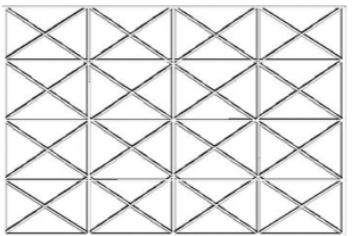

LBP

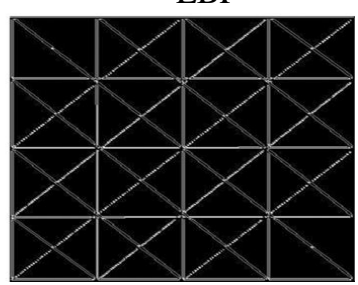

$2^{\text {nd }}$ order LDP in 45

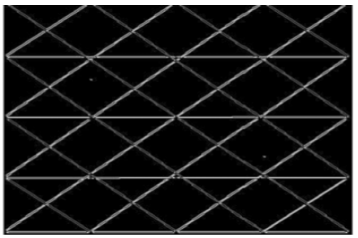

$2^{\text {nd }}$ order LDP in $90^{\circ}$

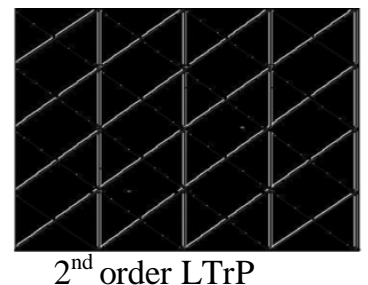

$2^{\text {nd }}$ order LTrP

Direction 1

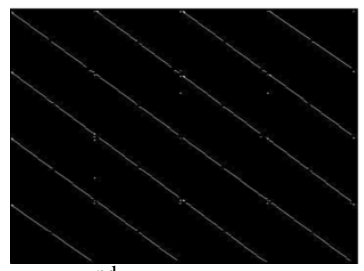

$2^{\text {nd }}$ order LTrP

Direction 3

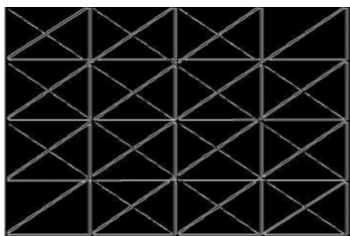

$2^{\text {nd }}$ order LDP in $135^{\circ}$
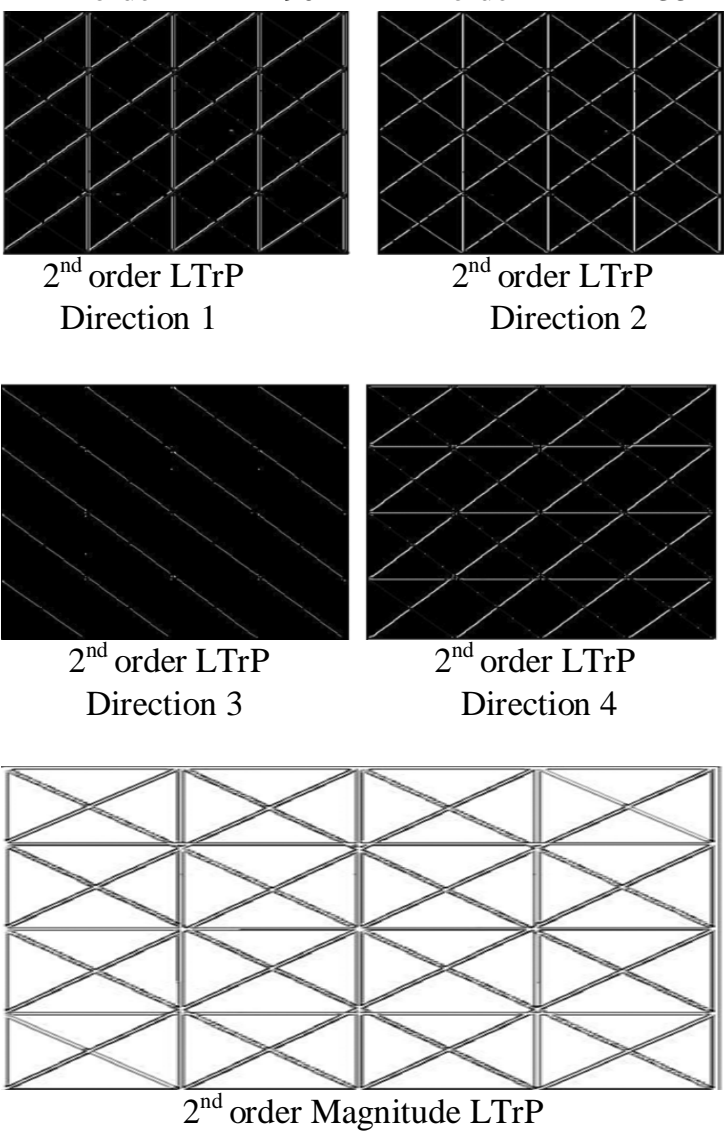

Figure 6: Image representations

Each pixel of the original image is considered as the center pixel, and it is coded by using the LBP, LTP, LDP, and LTrP descriptors with the help of neighbors. The possible values of various descriptors are in between 0 to 255 , which are further used for dimension reduction by uniform patterns. The LTrP extracts additional directional information, as compared with other patterns.

Figure 6 illustrates the results obtained by applying the LBP, LTP, LDP, and LTrP operators on a reference image. The reference image has been chosen since it provides the results that are visibly comprehensible to differentiate the effectiveness of these approaches. It can be observed that the second-order LTrP operator is able to extract detailed directional information, as compared with second-order LDP, LBP, and LTP operators. 
A.Usha Ruby et al., International Journal of Emerging Trends in Engineering Research, 8(4), April 2020, 956 - 963

\section{ADVANTAGES OF THE LTrP OVER OTHER PATTERNS}

The advantages of the LTrP over the LBP, the LDP, and the LTP can be justified with the help of three points.

1) The LBP, the LDP, and the LTP are able to encode images with only two (either "0" or " 1 "), two (either "0" or "1"), and three ("0," "1," or "-1") distinct values, respectively. However, the LTrP is able to encode images with four distinct values as it is able to extract more detailed information.

2) The LBP and the LTP encode the relationship between the gray value of the center pixel and its neighbors, whereas the LTrP encodes the relationship between the center pixel and its neighbors based on directions that are calculated with the help of (n-1)th-order derivatives.

3) The LDP encodes the relationship between the (n-1)thorder derivatives of the center pixel and its neighbors in $0^{\circ}$, $45^{\circ}, 90^{\circ}$, and $135^{\circ}$ directions separately, whereas the LTrP encodes the relationship based on the direction of the center pixel and its neighbors, which are calculated by combining (n-1)th-order derivatives of the $0^{\circ}$ and $90^{\circ}$ directions.

\section{IMAGE NORMALIZATION AND PREPROCESSING}

For face recognition, images from a database are in a generally normalized size by cropping images according to the location of eyes to eliminate the impact of the background. Moreover, pre-processing methods are often used to decrease noise factors, such as $\gamma$ correction and the Different of Gaussian (DoG) filter, which are believed to be the preferable methods for this problem. $\gamma$ correction is a nonlinear gray-level transformation that replaces gray-level I with $\mathrm{I} \gamma$ (for $\gamma>0$ ) or $\log (\mathrm{I})$ (for $\gamma=0$ ), where $\gamma$ is a userdefined parameter ranging from 0 to 1 , reflecting the performance of nonlinear transformation. $\gamma$ correction increases the contrast of images by enhancing the local dynamic ranges in the shadowed regions of the image while compressing them in bright regions. It is clear that the only factor in $\gamma$ correction is the value of $\gamma$ that is widely used in the range $[0,0.5]$.

To correct the uneven illumination, an image is first changed into the frequency domain by two-dimensional Fourier transform. Then it is filtered by the Different of Gaussian (DoG) filter to remove redundant information, while preserving the useful low-frequency information. The DoG filter is a common filter in the field of computer vision and image analysis, which consists of two Gaussian functions with different standard deviations. In the proposed method, the DoG with three scales $\mathrm{m}$ and two directions 0 and 90 are applied on a given image to compute derivatives in horizontal and vertical directions. The $\mathrm{n}^{\text {th }}$-order DoG LTrPs (DoGLTrPs) is obtained by performing the $(n-1)^{\text {th }}$ order derivatives.
Furthermore, the $12^{\text {th }}$ binary patterns per scale $\mathrm{m}$ are calculated similar to $11^{\text {th }}$ binary pattern, and the $13^{\text {th }}$ binary pattern is calculated similar to $12^{\text {th }}$ binary pattern.

Proposed System Framework

The algorithm for the proposed face recognition system is Input: Face Image; Output: Face Recognition

1. Load the image, and convert it into grayscale.

2. Apply the first-order derivatives in horizontal and vertical axis.

3. Calculate the direction for every pixel.

4. Divide the patterns into four parts based on the direction of the center pixel.

5. Calculate the tetra patterns, and separate them into three binary patterns.

6. Calculate the histograms of binary patterns.

7. Calculate the magnitudes of center pixels.

8. Construct the binary patterns, and calculate their histogram.

9. Combine the histograms calculated from steps 6 and 8 .

10. Construct the feature vector.

11. Compare the image with the images in the database.

12. Recognize the images based on the best matches.

This algorithm is also applied on Different of Gaussains for DoGLTrP. To correct the uneven illumination, an image is first changed into the frequency domain by twodimensional Fourier transform. Then it is filtered by the Different of Gaussian (DoG) filter to remove redundant information, while preserving the useful low-frequency information. The DoG filter consists of two Gaussian functions with different standard deviations

\section{EXPERIMENTAL RESULTS AND PERFORMANCE ANALYSIS}

In order to evaluate the feasibility and effectiveness of the proposed model, an extensive experimental investigation is conducted, covering face recognition under various situations including controlled condition, different lighting conditions, facial expressions, and partially obstructed conditions. The experiments were conducted on JAFFE, ORL, Essex, and YALE B databases which contain face images under all different conditions appropriate for face recognition. The closest match of the testing sample with any one of the training samples has been identified using DoG and such match is validated.

\section{DATABASE DESCRIPTION}

\subsection{ORL}

The ORL database contains a set of face images taken between 1992 and 1994 at the Ollivetti Research Laboratory 
in Cambridge. It contains 400 images of 40 subjects. Some images were captured at different times and have different variations including expression, lighting, and facial details (glasses/no glasses). The images were taken with a tolerance for some tilting and rotation of the face images up to $20^{\circ}$, and the image size is $92 \times 112$ pixels. These images are used to validate the performance of the texture model for face recognition under neutral conditions, facial expressions, illumination variation, and partially occluded conditions.

\subsection{YALE B}

The YALE B face database contains 5760 single light source images of 10 subjects each seen under 576 viewing conditions ( 9 poses X 64 illumination conditions). For every subject in a particular pose, an image with ambient (background) illumination was also captured. Hence, the total number of images is in fact $5760+90=5850$. These images are used to examine the performance of Dominant LTrP under different illuminations and pose variation conditions.

\section{EXPERIMENTAL RESULTS}

In this experiment, database ORL and Yale B is used. The performance of the proposed method is measured in terms of ARR computed using (30). Databases have been used to compare the performance of DLTrP with other existing methods (the LTP, the LDP, and the LBP) with and without DoG. From Table I, it is evident that the LTrP/DoGLTrP outperforms other existing methods. As shown in Figure 7 \& 8 , the DoGLTrPs produce a 59-dimensional feature vector less than the 256-dimensional feature vector produced by LTrPs.

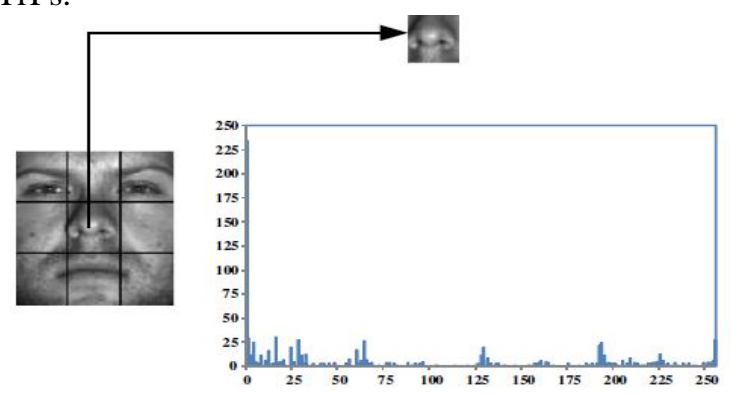

Figure 7: Histogram of LTrP

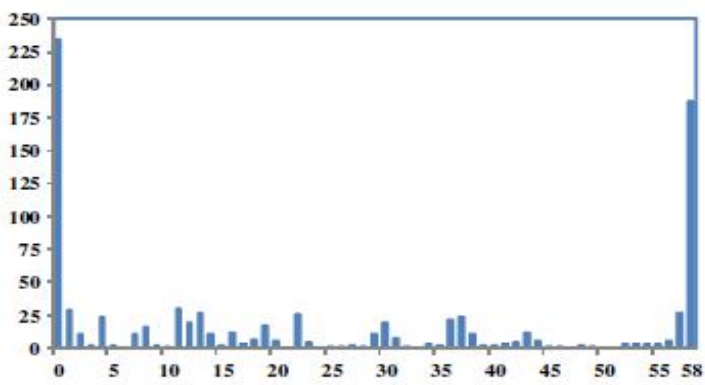

Figure 8: Histogram of DoGLTrP
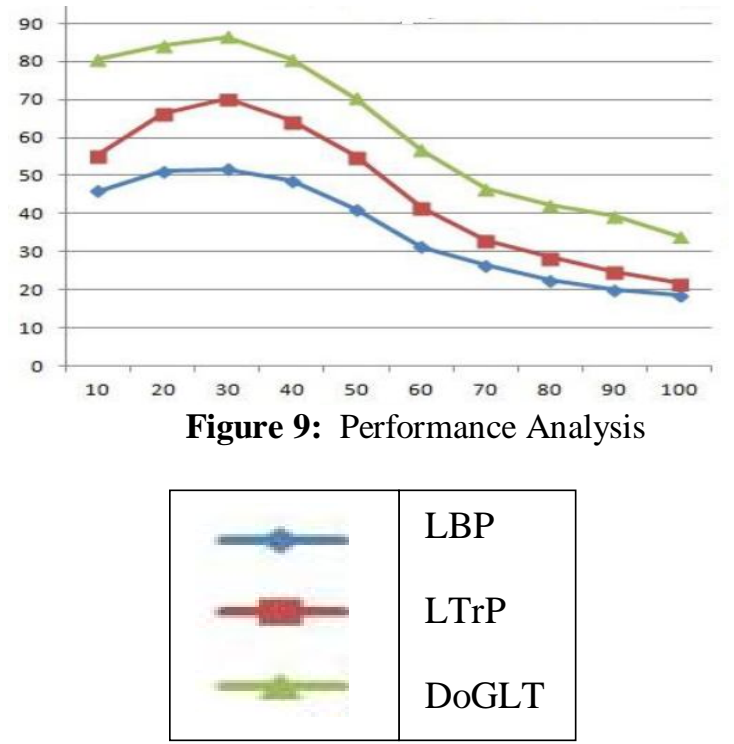

The performance of proposed face recognition is compared with Local Binary Pattern (LBP), LTrP and DoGLTrP. Based on this analysis which is shown in Figure 9, the proposed method is powerful and effective.

Table 1: ARR for the Face Classes of Yale B Database and ORL Database

\begin{tabular}{|c|c|c|}
\hline \multirow{2}{*}{ Method } & \multicolumn{2}{|c|}{$\operatorname{ARR}(\%)$} \\
\hline & Yale B & ORL \\
\hline DoGLTrP_2 $2^{\text {nd }}=$ Order & 86.11 & 91.01 \\
\hline DoGLTrP_3 $3^{\text {rd }}{ }_{-}$Order & 83.41 & 89.60 \\
\hline DoGLTrP $4^{\text {th }}-$ Order & 76.79 & 80.02 \\
\hline LTrP_ $2^{\text {nd }} \_$Order & 79.92 & 91.17 \\
\hline LTrP_3 $3^{\text {rd }}-$ Order & 79.47 & 85.49 \\
\hline LTrP_4 $4^{\text {th }}$ Order & 77.35 & 81.60 \\
\hline LDP_2 $2^{\text {nd }}-$ Order & 82.51 & 87.52 \\
\hline LDP_3 ${ }^{\text {rd }}$ Order & 79.98 & 82.24 \\
\hline LDP_4 $4^{\text {th }}$ Order & 77.34 & 81.79 \\
\hline LTP & 82.52 & 87.56 \\
\hline LBP & 79.98 & 82.24 \\
\hline GLTrP_2 $2^{\text {nd }} \_$Order & 81.96 & 83.41 \\
\hline GLTrP_3 $3^{\text {rd }}$ Order & 82.04 & 88.20 \\
\hline GLTrP_4 $4^{\text {th }}$ Order & 81.53 & 87.33 \\
\hline GLDP_2 ${ }^{\text {nd }}=$ Order & 79.12 & 87.53 \\
\hline GLDP_3 ${ }^{\text {rd }}{ }_{-}$Order & 79.23 & 88.19 \\
\hline GLDP_4 $4^{\text {th }}$ Order & 78.89 & 87.62 \\
\hline
\end{tabular}

The performance of the proposed method is measured in terms of ARR is given

$$
\mathrm{ARR}=\frac{1}{|D B|} \sum_{i=1}^{|D D|} R\left(I_{i}, \mathrm{n}\right)
$$

Table 1 shows the results of all techniques with and without DoG in terms of ARR. It is evident that the LTrP/DoGLTrP outperforms other existing methods. 


\section{CONCLUSION AND FUTURE WORK}

In this paper, we have presented a novel approach referred as LTrP for face recognition. The LTrP encodes the images based on the direction of pixels that are calculated by horizontal and vertical derivatives. The magnitude of the binary pattern is collected using magnitudes of derivatives. The effectiveness of the proposed approach has been also analysed by combining it with the DoG. The performance is measured in terms of ARR. using Yale B and ORL databases the result shows that DLTrP outperforms LTP, LDP, LBP and LTrP.

\section{REFERENCES}

1.Zhao,W. and Chellappa,R. and Phillips,P.J. and Rosenfeld,A. Face recognition: A literature survey, ACM Computing, Volume: 35, Issue: 04, Pages: 399 - 458,2013. https://doi.org/10.1145/954339.954342

2.Ding,X.Q. and Fang.C. Discussions on some problems in face recognition, Advances in Biometric Person Authentication, Proceedings, Lecture Notes in Computer Science: Springer Berlin/Heidelberg, Volume: 3338, Pages $47-56,2005$.

https://doi.org/10.1007/978-3-540-30548-4_7

3.Xiaoyang Tan, Songcan Chen, Zhi-Hua Zhou, Fuyan Zhang. Face recognition from a single image per person: A survey, Pattern Recognition : Elsevier, Volume: 39, Issue: 09, Pages: 1725 - 1745, 2006.

https://doi.org/10.1016/j.patcog.2006.03.013

4.Zhenhua Guo, Shenzhen, Zhang.D. A Completed Modeling of Local Binary Pattern Operator for Texture Classification, Image Processing, IEEE Transactions, Volume: 19 , Issue: 6, Pages: 1657 - 1663, March 2010.

https://doi.org/10.1109/TIP.2010.2044957

5.A. Ahmadian and A. Mostafa. An efficient texture classification algorithm using gabor wavelet, in Proc. EMBS, Pages 930-933, 2003.

6.H. Abrishami Moghaddama, ,T. Taghizadeh Khajoieb, A.H. Rouhib, M. Saadatmand Tarzjana. Wavelet correlogram: A new approach for image indexing and retrieval, Pattern Recognition, Volume 38, Issue 12, Pages 2506-2518, December 2005.

https://doi.org/10.1016/j.patcog.2005.05.010

7.Pallavi. D. Wadkar, Jitendra .M.Bakliwal, Megha Wankhade. Discrete Wavelet Transform in face recognition, International Journal of Emerging trends \& technology in computer science, Volume 2, Issue 3, May 2013.

8.Linlin Shen, Li Bai. A review on gabor wavelets for face recognition, Pattern Analysis and Applications, Volume 9, Pages: 273 - 292, 2006. https://doi.org/10.1007/s10044-006-0033-y

9.Michael Lyons \& Shigeru Akamatsu. Coding facial expressions with gabor wavelets, Proceedings, Third IEEE
International Conference on Automatic face gesture recognition, April 1998, Pages: 200-205, 1998.

10.Hasan Demiral, Gholamreza Anbarjafan. Satellite image resolution enhancement using complex wavelet transform, IEEE geoscience and remote sensing letters, Volume: 7, Issue: 1, Pages: 123 - 126, January 2010.

https://doi.org/10.1109/LGRS.2009.2028440

11.Baochang Zhang, Yougsheng Gao, Sanqiang Zhao, Jianzhuang Liu. Local derivative pattern versus local binary pattern : face recognition with high order local pattern description, IEEE transactions on image processing, Volume: 19, Issue: 2, Pages: 533 - 544, February 2010.

https://doi.org/10.1109/TIP.2009.2035882

12.Jianfens Ren, Xudong Jians, Junsong Yuan. Relaxed Local Ternary Pattern face recognition, IEEE International conference in Image Processing, September 2013. 